\title{
Dihydroartemisinin inhibits the Raf/ERK/MEK and PI3K/AKT pathways in glioma cells
}

\author{
WEI DU $^{1 *}$, CHANGHE PANG $^{1 *}$, YAKE XUE $^{1}$, QINGJUN ZHANG ${ }^{2}$ and XINTING WEI ${ }^{1}$ \\ ${ }^{1}$ Department of Neurosurgery, The First Affiliated Hospital, Zhengzhou University, Zhengzhou, Henan 450052; \\ ${ }^{2}$ Department of Neurosurgery, Peking University People's Hospital, Beijing 100044, P.R. China
}

Received June 2, 2014; Accepted July 30, 2015

DOI: 10.3892/ol.2015.3699

\begin{abstract}
It has previously been reported that dihydroartemisinin (DHA) is an effective novel anticancer compound in a number of types of tumor cells. Previous studies have demonstrated the anticancer activity of DHA in gioma cells. However, its underlining mechanism remains unclear. In the present study, the anticancer activity of DHA was examined in the glioma cell lines BT325 and C6. Western blot analysis was also employed to determine the signaling pathway changes. It was demonstrated that DHA effectively inhibited cell growth and induced apoptosis in glioma cells. Moreover, western blot analysis indicated that DHA-induced apoptosis was accompanied by inactivation of the Raf/MEK/ERK and PI3K/AKT signaling pathways, in addition to the downregulation of anti-apoptotic proteins Mcl-1 and Bcl-2 expression levels.
\end{abstract}

\section{Introduction}

Glioma represents the most prevalent and lethal primary brain tumor (1). A number of glioma cases have demonstrated low responses to conventional cytotoxic chemotherapy. Despite great efforts to improve therapeutics, the clinical outcome of glioma remains poor (1). Therefore, there is an urgent need to develop novel treatment to improve the prognosis of glioma (2).

Correspondence to: Professor Xinting Wei, Department of Neurosurgery, The First Affiliated Hospital, Zhengzhou University, 1 East Jian Road, Zhengzhou, Henan 450052, P.R. China

E-mail: weixinting777@163.com

${ }^{*}$ Contributed equally

Abbreviations: DHA, dihydroartemisinin; MTT, 3-(4,5-dimethyl-thiazol-2-yl)-2,5-diphenyl tetrazolium bromide; MEK, mitogen-activated and extracellular signal-regulated kinase kinase); ERK, extracellular signal-regulated kinase; MAPK, mitogen-activated protein kinase; PI3K, phosphatidylinositol 3-kinase; PIP3, phosphatidylinositol 3,4,5 triphosphate

Key words: dihydroartemisinin, glioma, dual pathway inhibitor, Raf/MEK/ERK, PI3K/AKT
Dysregulated signaling pathways have been considered to be involved in cancer progression; the Raf/mitogen-activated and extracellular signal-regulated kinase kinase (MEK)/extracellular signal-regulated kinase (ERK) cascade and the phosphatidylinositol 3-kinase (PI3K)/Akt cascade are commonly activated pathways in a number of types of cancer (3).

The Raf/MEK/ERK pathway is one of the most common oncogenic pathways and is critical in driving cell proliferation, survival and preventing apoptosis. Upon activation by growth factors, serum, polypeptide hormones, neurotransmitters or chemokines, activated ERK phosphorylates and regulates multiple cytoplasmic signaling proteins and transcription factors (4). These events result in gene expression changes and alterations in cell survival, division and metabolism. A previous study demonstrated that dysregulation of this pathway is involved in the oncogenesis of various types of human cancer (5). Therefore, inhibition of the Raf/MEK/ERK pathway is a promising strategy for cancer therapy (6).

The PI3K/AKT pathway is another crucial signaling pathway for cancer development (7). Growth factors and cytokines activate the lipid kinase PI3K and then generate phosphatidylinositol 3,4,5-triphosphate (PIP3), which activates the recruitment of protein kinase AKT to cell membrane. Activated AKT phosphorylates Ser/Thr residues in proteins that are involved in cell survival, proliferation, and metabolic pathways. Abberrant AKT activation has been documented in a number of types of human cancer (8). Therefore, inhibition of the PI3K/AKT pathway is also a promising strategy for cancer therapy (9).

A previous study indicated that the Raf/MEK/ERK and PI3K/AKT signaling pathways cooperatively link with each other to enhance the proliferation and apoptotic resistance capacity in cancer cells (10). Both signaling pathways are activated by growth factor receptors and their complicated crosstalk at multiple points greatly increases the therapeutic effects of single signaling inhibition in cancer cells (11). In addition, these two pathways can exert complementary and redundant functions when only one single pathway is inhibited (12). Therefore, more clinical benefit may be obtained by simultaneously targeting both cascades (13).

Dihydroartemisinin (DHA) is a semi-synthetic derivative of the anti-malarial drug artemisinin. Previous studies have demonstrated that DHA may exert its anti-proliferation and 
apoptosis-inducing effects on a number of types of cancer cells, including leukemia, prostate cancer, ovarian cancer and colorectal cancer cells (14-17). In addition, it has been reported that DHA induces apoptosis in C6 glioma cells by increasing the generation of reactive oxygen species (ROS) and inhibiting activation of hypoxia inducible factor-1 $\alpha$ (HIF-1 $\alpha$ ) (18). However, the underlying molecular mechanisms by which DHA exerts its anti-glioma effects remain unclear. In the present study, the effects of DHA treatment of glioma cells and the potential molecular mechanisms were investigated, particularly focusing on the impact on Raf/MEK/ERK and PI3K/AKT pathways.

\section{Materials and methods}

Cells and reagents. The BT325 cell line was obtained from Beijing Neurosurgical Institute Collection (Beijing, China) and the C6 cell line was purchased from American Type Culture Collection (Manassas, VA, USA). All cells were cultured at $37^{\circ} \mathrm{C}$ in Dulbecco's modified Eagle medium (DMEM) (Gibco Life Technologies, Carlsbad, CA, USA) containing $10 \%$ fetal bovine serum (FBS) (Gibco), and $100 \mathrm{U} / \mathrm{ml}$ penicillin-streptomycin (Gibco). Dihydroartemisinin (DHA) was purchased from Sigma-Aldrich (St. Louis, MO, USA) and dissolved in dimethylsulfoxide (DMSO) with cell medium resulting in a final DMSO concentration of $1 \%$.

Cell viability assay. Cell viability was measured by 3-[4,5-dimethylthiazol-2-thiazolyl]-2,5-diphenyl-tetrazolium bromide (MTT) assay. Logarithmically growing glioma cells were seeded in 96-well culture clusters (Costar, Cambridge, MA, USA) at a density of 5,000-6,000 cells/well with culture medium and incubated for $24 \mathrm{~h}$. On the following day, cells were treated with desired concentrations of DHA. Four hours before the desired time points, $10 \mu \mathrm{l}$ of $10 \mathrm{mg} / \mathrm{ml}$ MTT was added, and incubated for $4 \mathrm{~h}$, the medium was removed and $200 \mu \mathrm{l}$ DMSO was added to each well. The optical density (OD) value at $570 \mathrm{~nm}$ was measured using MRX II absorbance reader (DYNEX Technologies, Chantilly, VA, USA). The cell viability was expressed as the percentage of absorbance in cells with DHA treatment versus the control group. The experiment was replicated in triplicate. Results were expressed as the mean \pm standard deviation (SD) values.

Apoptosis analysis by flow cytometry. Apoptotic cells were measured with Annexin V-fluorescein isothiocyanate (FITC)/propidium iodide (PI) kit (BD Biosciences, Sparks, MD, USA). Briefly, cells were cultured in 6-well plates at $3 \times 10^{5}$ cells per well. After DHA-treatment for $10 \mathrm{~h}$, a cell suspension was prepared by trypsinization, and was centrifuged at $800 \mathrm{x} \mathrm{g}$ for $5 \mathrm{~min}$ at $4^{\circ} \mathrm{C}$, and then the cells were resuspended in $500 \mu \mathrm{l}$ of binding buffer. The cells were incubated with $10 \mu \mathrm{l}$ Annexin V-FITC solution and $5 \mu \mathrm{l} \mathrm{PI}$ at $37^{\circ} \mathrm{C}$ for $30 \mathrm{~min}$ away from the light. FACs analysis was performed by flow cytometry (BD Biosciences, Franklin Lakes, NJ, USA). For each sample, 10,000 cells were analyzed. All of the experiments were performed in triplicate.

Western blot analysis. Cells were plated in tissue culture dishes overnight and treated with DHA at the given concentrations for $24 \mathrm{~h}$. Following harvest, adherent cells were washed with cold PBS and resuspended in lysis buffer $(150 \mathrm{mM} \mathrm{NaCl}$, $50 \mathrm{mM}$ Tris- $\mathrm{HCl}, \mathrm{pH}$ 7.4, 2 mM ethylenediaminetetra-acetic acid (EDTA), 1\% NP-40) containing protease inhibitor cocktail (Amresco Inc., Framingham, MA, USA). Protein levels in the extracts were quantified using BCA assay (Pierce Biotechnology, Inc., Rockford, IL, USA). Equal amounts of total protein extracts were resolved by $10 \%$ standard sodium dodecyl sulfate polyacrylamide gel electrophoresis (SDS-PAGE) and transferred onto a polyvinylidene fluoride (PVDF) membrane (0.45 mm; EMD Millipore, Billerica, MA, USA). Membranes were blocked with $5 \%$ fat-free dry milk/Tris-buffered saline (TBS)-Tween 20 (TBST; cat. no. 9997, Cell Signaling Technology Inc., Danvers, MA, USA) at room temperature for $1 \mathrm{~h}$, then incubated with the following anti-human primary antibodies to MEK (monoclonal rabbit; 1:1,000; cat. no. 8727), ERK (monoclonal rabbit; 1:1,000; cat. no. 4348), AKT (monoclonal mouse; 1:1,000; cat. no. 12694), phospho-MEK (monoclonal rabbit; 1:1,000; cat. no. 2338), phospho-ERK (monoclonal rabbit; 1:1,000; cat. no. 4377), phospho-AKT (polyclonal rabbit; 1:1,000; cat. no. 9272), Bcl-2 (monoclonal mouse; 1:500; cat. no. 15071), Mcl-1 (monoclonal rabbit; 1:500; cat. no. 5453), Bcl-xL (monoclonal rabbit; 1:500; cat. no. 13835), Bax (monoclonal rabbit; 1:500; cat. no. 5023) and GAPDH (monoclonal rabbit; 1:2,000; cat. no. 2118; Cell Signaling Technology Inc.) overnight at $4^{\circ} \mathrm{C}$. The membranes were then washed three times with TBST for $5 \mathrm{~min}$ at room temperature. Secondary horseradish peroxidase-linked mouse anti-sheep $(1: 1,000$; cat. no. 7076$)$ or rabbit anti-sheep IgG (1:1,000; cat. no. 5127) antibodies (Cell Signaling Technology Inc.) were incubated for $1 \mathrm{~h}$ at room temperature. The membranes were then washed three times with TBST for $5 \mathrm{~min}$ at room temperature, and the immunoblots were visualized by enhanced chemiluminescence reagent (GE Healthcare Life Sciences, Logan, UT, USA).

Statistical analysis. Data are expressed as the mean \pm SD, and evaluated for significant differences using one-way analysis of variance (ANOVA) via SPSS software, version 13.0 (SPSS Inc., Chicago, IL, USA). A value of $\mathrm{P}<0.05$ was considered to indicate a statistically significant difference.

\section{Results}

DHA inhibited glioma cell proliferation. The effects of DHA on inhibition of proliferation of BT325 and C6 cells at dosages between 25-200 $\mu \mathrm{M}$, were examined. MTT assay demonstrated that the effects of DHA on the viability of the cell lines were dose- and time-dependent (Fig. 1). Treatment of BT325 and C6 cells with $100 \mu \mathrm{M}$ DHA for $48 \mathrm{~h}$ caused $62.5 \pm 3.6 \%$ and $77.3 \pm 6.4 \%$ reduction in cell viability, respectively (Fig. 1B; $\mathrm{P}<0.05)$.

DHA results in apoptotic death in glioma cells. The proapoptotic effects of DHA were examined in BT325 and C6 cells. As presented in Fig. 2, DHA promoted apoptosis in both cell lines in a dose-dependent manner $(\mathrm{P}<0.05$; Fig $2 \mathrm{C}$ and $\mathrm{D})$.

DHA inhibits Raf/MEK/ERK signaling pathway in gliomacells. A previous study reported that DHA inhibits Raf/MEK/ERK 

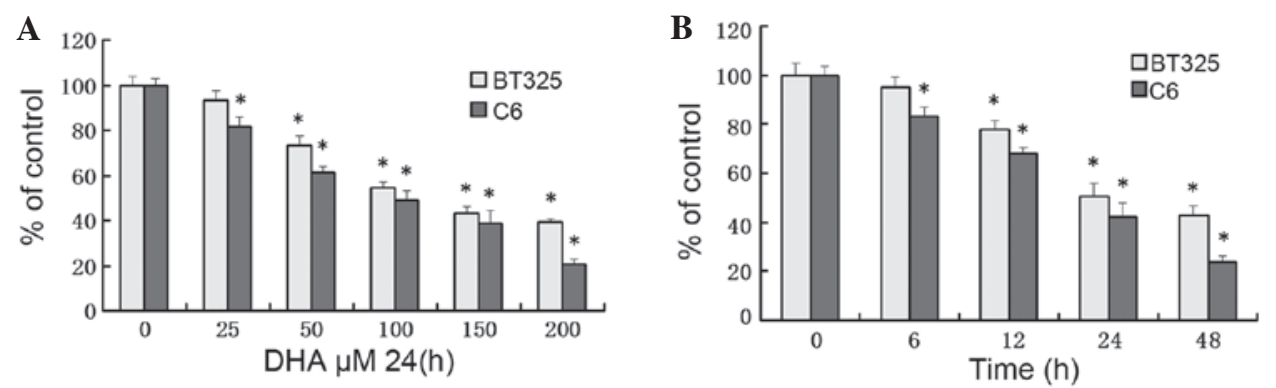

Figure 1. Effect of DHA on cell viability of BT325 and C6 cells. (A) Logarithmically growing BT325 and C6 cell lines were incubated with different concentrations of DHA for $24 \mathrm{~h}$. (B) BT325 and C6 cell lines were incubated with $100 \mu \mathrm{M}$ DHA for 6, 12, $24 \mathrm{and} 48 \mathrm{~h}$. At the end of incubation, the cell survival rates were determined by MTT assay. Cell viability is expressed as the percentage of cell survival compared with the control. Data are from 3 independent experiments. ${ }^{*} \mathrm{P}<0.05$, compared with the control group.

A

\section{ВТ325}

$0 \mu \mathrm{M}$

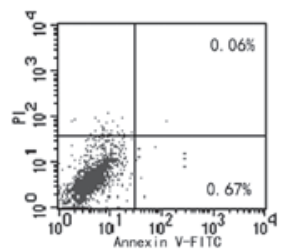

B

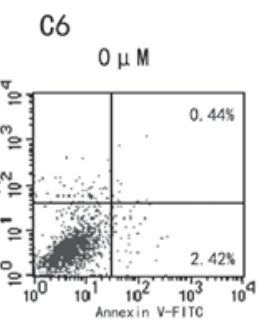

C
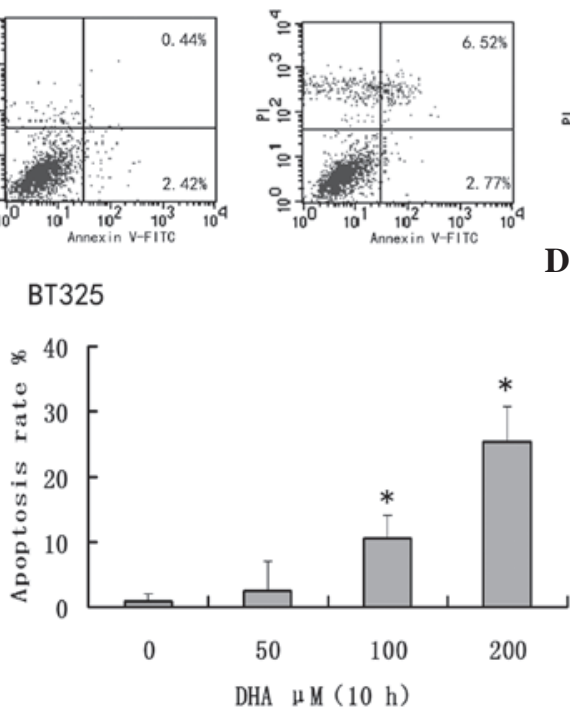

D
$100 \mu \mathrm{M}$

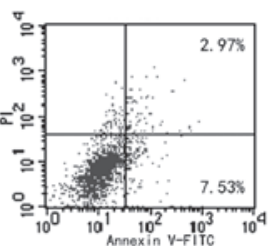

$100 \mu \mathrm{M}$

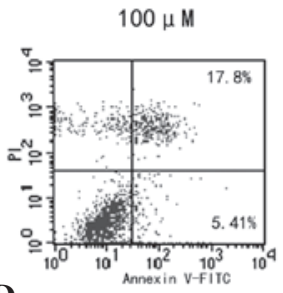

C6

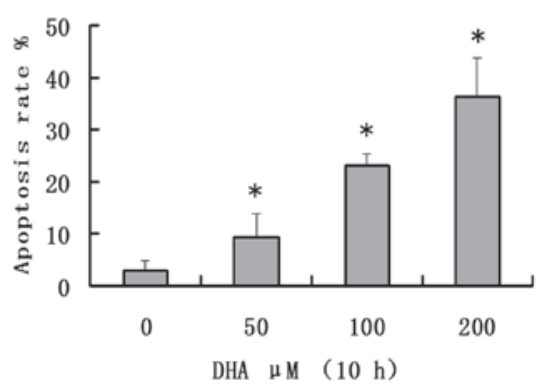

Figure 2. DHA induces apoptosis in BT325 and C6 cells. Logarithmically growing BT325 and C6 cells were incubated with different concentrations of DHA for $10 \mathrm{~h}$. Cells were collected and stained with Annexin V/PI followed by FACS analysis. FACS plots demonstrating apoptotic rates in (A) BT325 and (B) C6 cells. The four different cell populations marked as: unstained cells indicating live cell population (LL, lower left), Annexin V positive and PI negative stained cells indicating early apoptosis (LR, lower right), Annexin V/PI double-stained cells showing late apoptosis (UR, upper right), and Annexin V negative and PI positive stained cells showing dead cells (UL, upper left). Apoptosis was defined as Annexin V staining positive. Graphs demonstrating quantification of apoptosis in (C) BT325 and (D) C6 cells. ${ }^{*} \mathrm{P}<0.05$ vs. control group ( $0 \mu \mathrm{M}$ DHA).

pathway in a number of types of cancer (14). Therefore the impact on the phosphorylation of these signaling molecules was examined in glioma cells. Western blot analysis demonstrated that after $24 \mathrm{~h}$ of DHA exposure, the expression levels of Raf/MEK/ERK signaling pathway members were reduced in both cell lines in a dose-dependent manner (Fig. 3).

DHA inhibits AKT activation in glioma cells. Dysregulation of the PI3K/AKT pathway is commonly observed in glioma cells (19). The effects of DHA on the activation of PI3K/AKT signaling were examined in glioma cells. Western blot analysis demonstrated that after $24 \mathrm{~h}$ of DHA exposure, DHA treatment markedly inhibited the phophorylation of AKT in a dose-dependent manner (Fig. 4).

Involvement of Bcl-2 family proteins in DHA-induced glioma cell apoptosis. The Bcl-2 family proteins serve important roles in the regulation of cell apoptosis (20). The effects of DHA 


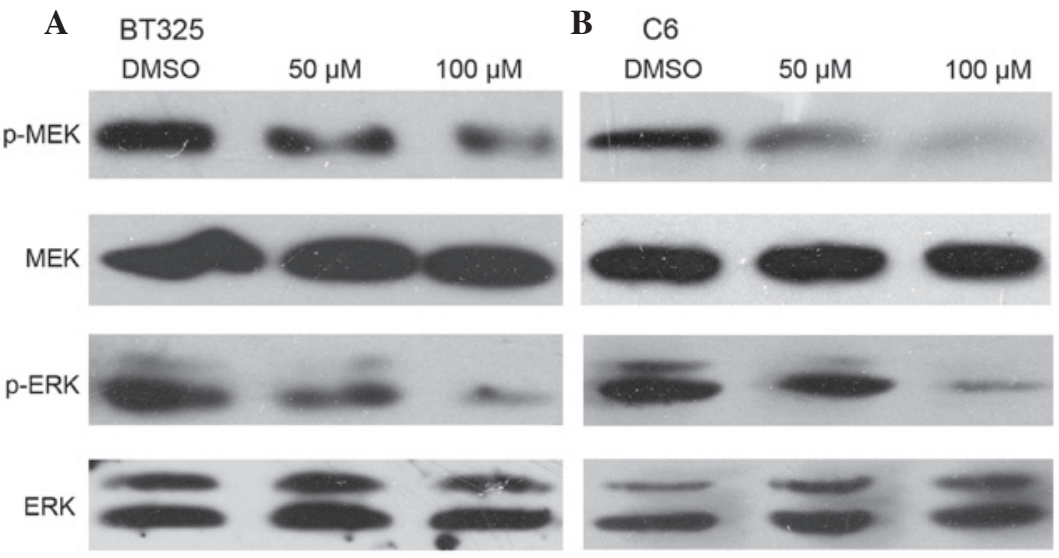

Figure 3. DHA inhibits RAF/MEK/ERK and PI3K/AKT signaling pathways in (A) BT325 and (B) C6 cells. Cells were treated with the indicated concentrations of DHA for $24 \mathrm{~h}$. Western blot analysis was performed using specific antibodies against the indicated proteins.
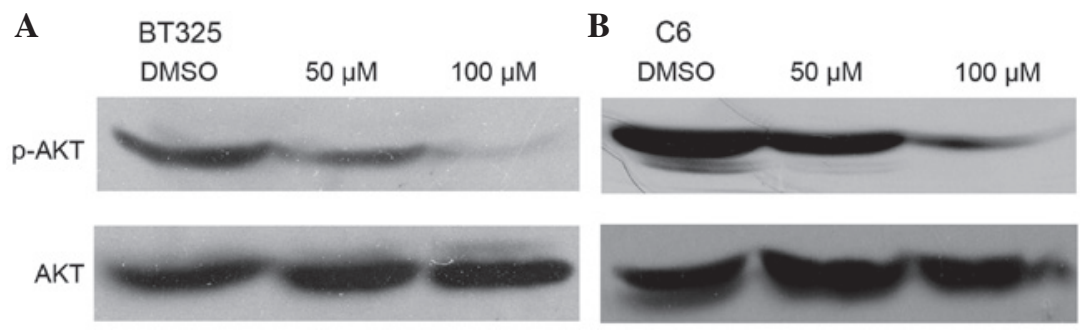

Figure 4. DHA inhibits PI3K/AKT signaling pathway in (A) BT325 and (B) C6 cells. Cells were treated with the indicated concentrations of DHA for $24 \mathrm{~h}$.

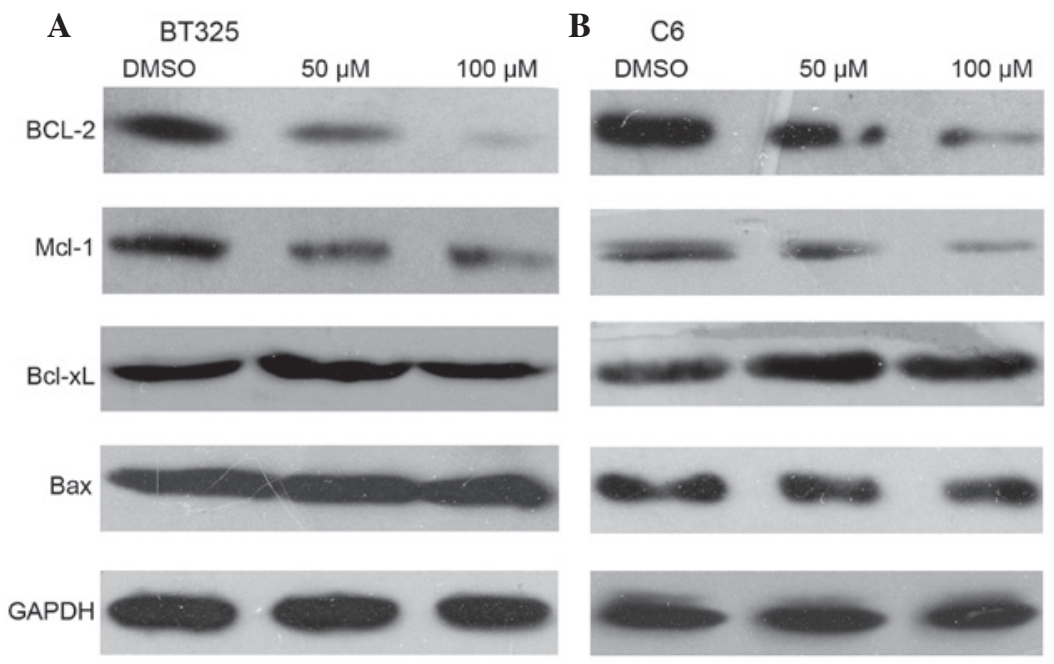

Figure 5. DHA downregulates Bcl-2 and Mcl-1 protein expression levels in (A) BT325 and (B) C6 cells, but has no marked effect on protein expression of $\mathrm{Bcl}-\mathrm{xL}$ or Bax. The cells were harvested after incubation with the indicated concentrations of DHA for $24 \mathrm{~h}$. GAPDH was used as an internal control.

treatment on Bcl-2 protein expression levels were examined. Western blot analysis demonstrated that DHA treatment markedly inhibited the expression of Bcl-2 and Mcl-1, two antiapoptotic Bcl-2 proteins (Fig. 5). However, DHA treatment had no impact on the protein expression levels of Bcl-xL or Bax.

\section{Discussion}

As important anti-apoptotic signaling pathways, Raf/MEK/ERK and PI3K/AKT signaling cascades have attracted considerable attention (3). These signaling pathways have been demonstrated to serve critical roles in the transduction of growth factor signals, which regulate gene expression to control cell survival, proliferation, motility, and metabolism. Mutations may occur in upstream receptors such as EGFR and Flt-3, or downstream members such as Ras, Raf, PI3K, PTEN and AKT (13). Accumulating evidence has indicated that the crosstalk between Ras/Raf/MEK/ERK and the PI3K/AKT pathways serves important roles in promoting cell proliferation and inhibiting apoptosis of cancer, and pathway cross-inhibition reduces the effectiveness of single agents (12). A number of studies have demonstrated synergistic anticancer effects of simultaneous inhibition of both pathways. Treatment 
of basal-like cell lines with a selective MEK inhibitor resulted in enhanced PI3K pathway signaling, whereas supplementation of PI3K inhibitor induces apoptosis both in vitro and in vivo $(18,21)$. These evidence indicates that dual inhibition of Raf/MEK/ERK and PI3K/AKT signaling may result in more clinical benefit and therefore may provide certain advantages over single pathway inhibitors (22).

Although the anti-cancer activity of DHA has been demonstrated in a number of types of cancer (23-25), the underlying mechanisms remain largely unknown. To optimize the therapeutic effect of DHA against glioma cells and to explore its molecular mechanisms, the BT325 and C6 cell lines were used in the present study. Indeed, using cell viability and apoptosis analysis, it was indicated that DHA is capable of inducing the apoptosis of human glioma cells in a concentration- and time-dependent manner. For further illuminating the possible mechanisms, the present study was extended to investigate the effect of DHA on MEK/ERK and PI3K/AKT signaling factors. The results demonstrated that DHA suppressed MEK, ERK and AKT phosphorylation in the dose-dependent manner, indicating that DHA may induce apoptosis through a process that involves Raf/MEK/ERK and PI3K/AKT pathways inactivation. The Raf/MEK/ERK and PI3K/AKT signal pathways have been implicated in a wide variety of processes in cancer cells including the regulation of cell proliferation, survival and apoptosis. Therefore, the present study hypothesized that the combination of Raf/MEK/ERK and PI3K/AKT pathway inhibition and DHA treatment would greatly suppress the proliferation of glioma cells and markedly induce apoptosis.

Inducing apoptosis is an attractive strategy for cancer therapy, which is finally determined by the balance between pro- and anti-apoptotic mechanisms (20). The expression of the $\mathrm{Bcl}-2$ protein family was also examined; this family of proteins serves important roles in the regulation of mitochondria-dependent apoptosis. Bcl-2 protein is the prototype of this family, which inhibits cell apoptosis through multiple mechanisms (26). Mcl-1 is another highly expressed anti-apoptotic protein expressed in a number of types of malignancies and has been demonstrated to mediate resistance to chemotherapy, whereas Bax is a pro-apoptotic member. In the present study, no significant differences in the expression of Bax protein were observed after DHA treatment in glioma cells. However, the expression of Bcl-2 and Mcl-1 proteins was significantly suppressed by DHA in a dose-dependent manner. These results indicated that DHA may exerts its anti-glioma effects through the inhibition of pro-apoptotic proteins Bcl-2 and Mcl-1.

In summary, the data in the present study indicates that DHA suppresses the Raf/MEK/ERK and PI3K/AKT pathways in the glioma cells, which provides valuable information on the molecular mechanism of its anticancer activity. Therefore, DHA may be a promising molecule for the treatment of glioma.

\section{References}

1. Sathornsumetee S, Reardon DA, Desjardins A, et al: Molecularly targeted therapy for malignant glioma. Cancer 110: 13-24, 2007.

2. Wang Y and Jiang T: Understanding high grade glioma: Molecular mechanism, therapy and comprehensive management Cancer Lett 331: 139-146, 2013.

3. Saini KS, Loi S, de Azambuja E, et al: Targeting the PI3K/AKT/mTOR and Raf/MEK/ERK pathways in the treatment of breast cancer. Cancer Treat Rev 39: 935-946, 2013.
4. Redman EK, Brookes PS and Karcz MK: Role of p90(RSK) in regulating the crabtree effect: implications for cancer. Biochem Soc Trans 41: 124-126, 2013.

5. Roberts PJ and Der CJ: Targeting the Raf-MEK-ERK mitogen-activated protein kinase cascade for the treatment of cancer. Oncogene 26: 3291-3310, 2007.

6. Hasskarl J: Sorafenib: Targeting multiple tyrosine kinases in cancer. Recent Results Cancer Res 201: 145-164, 2014.

7. Han L, Yang Y, Yue X, et al: Inactivation of PI3K/AKT signaling inhibits glioma cell growth through modulation of beta-catenin-mediated transcription. Brain Res 1366: 9-17, 2010.

8. Chin YR and Toker A: Function of Akt/PKB signaling to cell motility, invasion and the tumor stroma in cancer. Cell Signal 21: 470-476, 2009.

9. Ghayad SE and Cohen PA: Inhibitors of the PI3K/Akt/mTOR pathway: New hope for breast cancer patients. Recent Pat Anticancer Drug Discov 5: 29-57, 2010.

10. Li Q, Wu J, Zheng H, et al: Discovery of 3-(2-aminoethyl)-5-(3 -phenyl-propylidene)-thiazolidine-2,4-dione as a dual inhibitor of the Raf/MEK/ERK and the PI3K/Akt signaling pathways. Bioorg Med Chem Lett 20: 4526-4530, 2010.

11. Steelman LS, Abrams SL, Whelan J, et al: Contributions of the Raf/MEK/ERK, PI3K/PTEN/Akt/mTOR and Jak/STAT pathways to leukemia. Leukemia 22: 686-707, 2008.

12. Mendoza MC,Er EE and Blenis J: The Ras-ERK and PI3K-mTOR pathways: cross-talk and compensation. Trends Biochem Sci 36: 320-328, 2011.

13. McCubrey JA, Steelman LS, Kempf CR, et al: Therapeutic resistance resulting from mutations in Raf/MEK/ERK and PI3K/PTEN/Akt/mTOR signaling pathways. J Cell Physiol 226: 2762-2781, 2011.

14. Lee J, Zhang G, Wu X, Xu F, Zhou J and Zhang X: Growth inhibitory effect of dihydroartemisinin on Bcr/Abl+ chronic myeloid leukemia K562 cells involve AKT, ERK and NF-kappaB modulation. J Cancer Res Clin Oncol 138: 2095-2102, 2012.

15. Wang XM, Zhang L, Ding GF and Wang QZ: Inhibitory effect of dihydroartemisinin on the growth of human prostate cancer PC-3 M cells and its mechanism. Zhonghua Nan Ke Xue 18: 590-594, 2012 (In Chinese).

16. Feng X, Li L, Jiang H, Jiang K, Jin Y and Zheng J: Dihydroartemisinin potentiates the anticancer effect of cisplatin via mTOR inhibition in cisplatin-resistant ovarian cancer cells: involvement of apoptosis and autophagy. Biochem Biophys Res Commun 444: 376-381, 2014.

17. Lu M, Sun L, Zhou J and Yang J: Dihydroartemisinin induces apoptosis in colorectal cancer cells through the mitochondria-dependent pathway. Tumour Bio 35: 5307-5314, 2014.

18. Hoeflich KP, O'Brien C, Boyd Z, et al: In vivo antitumor activity of MEK and phosphatidylinositol 3-kinase inhibitors in basal-like breast cancer models. Clin Cancer Res 15: 4649-4664, 2009.

19. Kubiatowski T, Jang T, Lachyankar MB, Salmonsen R, Nabi RR, Quesenberry PJ, Litofsky NS, Ross AH and Recht LD: Association of increased phosphatidylinositol 3-kinase signaling with increased invasiveness and gelatinase activity in malignant gliomas. J Neurosurg 95: 480-488, 2001.

20. Yip KW and Reed JC: Bcl-2 family proteins and cancer. Oncogene 27: 6398-6406, 2008.

21. Mirzoeva OK, Das D, Heiser LM, et al: Basal subtype and MAPK/ERK kinase (MEK)-phosphoinositide 3-kinase feedback signaling determine susceptibility of breast cancer cells to MEK inhibition. Cancer Res 69: 565-572, 2009.

22. Sheppard KE, Cullinane C, Hannan KM, et al: Synergistic inhibition of ovarian cancer cell growth by combining selective $\mathrm{PI} 3 \mathrm{~K} / \mathrm{mTOR}$ and RAS/ERK pathway inhibitors. Eur J Cancer 49: 3936-3944, 2013

23. Huang XJ, Li CT, Zhang WP, Lu YB, Fang SH and Wei EQ: Dihydroartemisinin potentiates the cytotoxic effect of temozolomide in rat C6 glioma cells. Pharmacology 82: 1-9, 2008.

24. Lu YY, Chen TS, Qu JL, Pan WL, Sun L and Wei XB: Dihydroartemisinin (DHA) induces caspase-3-dependent apoptosis in human lung adenocarcinoma ASTC-a-1 cells. J Biomed Sci 16: $16,2009$.

25. Liu Y, Wang W, Xu J, et al: Dihydroartemisinin inhibits tumor growth of human osteosarcoma cells by suppressing Wnt/beta-catenin signaling. Oncol Rep 30: 1723-1730, 2013.

26. Heath-Engel HM, Chang NC and Shore GC: The endoplasmic reticulum in apoptosis and autophagy: Role of the BCL-2 protein family. Oncogene 27: 6419-6433, 2008. 